\title{
PRECISION VS. ACCURACY IN STAR CATALOGS
}

\author{
L. G. TAFF, J. E. MORRISON, AND R. L. SMART \\ STScI, 3700 San Martin Drive, Baltimore, MD 21218 USA
}

As better precision is achieved and more sophisticated reduction methods are created previously invisible biases surface. This has been especially true in astrometric Schmidt plate work. The problem of their amelioration is not fully solved and precision per se is meaningless in the presence of poor accuracy of comparable amplitude. Continuing to benignly neglect this issue puts us in the position of standing on only one statistical leg. New techniques have been designed to further minimize systematic errors. Of especial interest to star catalog analysis is the method of infinitely overlapping circles (Taff, Bucciarelli \& Lattanzi, ApJ 361, 667, 1990; Taff, Bucciarelli \& Lattanzi, ApJ 392, 746 1992; Bucciarelli, Taff \& Lattanzi, J. Stat. Comp. and Sim. 48, 29 1993). With it almost complete success has occurred with regard to the removal of systematic errors which creep into compilation catalogs as a result of inadequate treatment of catalog-to-catalog systematic errors; they can essentially be eliminated a priori or a posteriori (Bucciarelli, Lattanzi \& Taff, in press in ApJ 1994; Taff \& Bucciarelli, in press in ApJ 1994). What infinitely overlapping circles does can be briefly described as follows: Let $X(x)$ be the measured (true) value of a standard coordinate, $S(x, y)(\varepsilon)$ be the systematic (random) error in $x$ at this point, let $w_{m}$ be the infinitely overlapping circle weight, $\sigma$ be the standard deviation of the random error in $x, N$ be the total number of stars in this circle which has radius $R$, and $x_{0}, y_{0}$ be the coordinates of the center of this circle. Then, the relation between the measured value $X$ and the true value $x$ is given by

$$
X=x+S(x, y)+\varepsilon .
$$

Expanding $S$ in a Taylor series centered at $\left(\mathrm{x}_{0}, \mathrm{y}_{0}\right)$ yields---r $=(\mathrm{x}, \mathrm{y})--$

$$
\begin{aligned}
X=x+ & S\left(x_{0}, y_{0}\right)+\nabla S\left(x_{0}, y_{0}\right) \cdot\left(\mathbf{r}-r_{0}\right) \\
& +1 / 2\left(\mathbf{r}-\mathbf{r}_{0}\right) \cdot \nabla \cdot \nabla S\left(x_{0}, y_{0}\right) \cdot\left(\mathbf{r}-\mathbf{r}_{0}\right)+\text { higher order terms }+\varepsilon .
\end{aligned}
$$

Now, multiply this expression by the infinitely overlapping weight and integrate over the circle. Using the assumption that the stars are uniformly distributed over the circle and the intrinsic symmetry of the circle itself yields

$$
\begin{aligned}
\int_{(X-x) w_{-} d A} & =S\left(x_{0}, y_{0}\right)+\int_{\varepsilon w_{-}} d A+1 / 2 \nabla^{2} S\left(x_{0}, y_{0}\right)<R^{2}>+ \text { quartic terms } \\
& \sim S\left(x_{0}, y_{0}\right)+\sigma / N^{1 / 2}+1 / 2 \nabla^{2} S\left(x_{0}, y_{0}\right)<R^{2}>+ \text { quartic terms. }
\end{aligned}
$$

Thus, the bias at the center of the circle is recovered, the random errors are diminished, and the next term is only the Laplacian of the systematic errors.

Research supported by NASA Contract NAS5-32496. 